D.T. Wickramasinghe, L. Ferrario, and G.V. Bicknell, eds.

\title{
Compact Radio Cores in the Galactic Center and Elsewhere
}

\author{
Heino Falcke \\ Department of Astronomy, University of Maryland, College Park, MD \\ 20742-2421, USA
}

\begin{abstract}
Compact radio cores are not only common in radio galaxies and quasars but also in many nearby galaxies with low-active, supermassive black holes. One famous example is the Galactic Center source Sgr $\mathrm{A}^{*}$. Recent studies of proper motions and radial velocities of stars in the inner parsec of the Galaxy convincingly demonstrate the presence of a compact dark mass of $2.5 \cdot 10^{6} M_{\odot}$ in the nucleus of the Milky Way. Millimeter VLBI and submm observations of Sgr A* thus probe a region of only a few Schwarzschild radii in diameter. In this paper I will review our current theoretical and observational knowledge of this source and compare it to some famous LINER galaxies like NGC 4258, NGC 3079 , and NGC 6500. In all cases these radio cores can be well explained by a standard AGN jet model, and, with the exception of Sgr A*, large scale outflows are observed that have powers comparable to those inferred from the radio cores. Recent VLBI observations of radio-weak quasars and HST observations of Seyfert galaxies indicate that these AGN also produce powerful jets which, however, have relatively less luminous radio cores than radio-loud quasars and the LINERs discussed here. Therefore, jets and compact radio cores appear to be natural constituents of an AGN, but the reason why apparently some jets are radio-loud and others not remains a mystery.
\end{abstract}

\section{Introduction}

When talking about compact radio cores the first thing that comes to ones mind are usually the flat-spectrum radio cores in quasars and radiogalaxies to which a substantial fraction of VLBI time and many theoretical papers have been dedicated. Those cores are generally believed to be the bases of relativistic radio jets produced by the central engine of an AGN. However, these cores are probably only the tip of an iceberg, as the largest number of AGN reside in lowactive and less prominent galactic nuclei. Large surveys (Wrobel \& Heeschen 1984, Roy et al. 1994, Slee et al. 1994, Sadler et al. 1995) have revealed that quite a substantial number of nearby galaxies also show compact radio cores in their nuclei. As the presence of radio cores seems to be correlated with optical emission lines (O'Connell \& Dressel 1978) and since one third of all galaxies show evidence for optical activity (Ho et al. 1995), we can expect to find compact radio cores almost everywhere in the universe. One of the most prominent examples of such a radio core is of course $\mathrm{Sgr} \mathrm{A}^{*}$ in the Galactic Center. 
While over the years quite a few observational programs have tackled compact radio nuclei in nearby galaxies and low-power AGN, there have been only a few detailed discussions of their physical nature. Therefore, in the first part of this paper I will summarize the current observational and theoretical status of Sgr A*, which will be basically an update of my earlier reviews (Falcke 1996a\&b), and then move on to discuss in detail examples of radio cores and jets in prominent nearby galaxies like NGC 4258 , NGC 3079 , or NGC 6500 . Finally, I want to briefly discuss recent findings for Seyfert galaxies and radio-weak quasars, which suggest that there jets play an even larger rôle than initially thought.

\section{Sgr $A^{*}$ - Observations}

\subsection{Mass}

The largest progress in the Galactic Center has been made in the determination of the mass of Sgr A*. Almost one decade ago Genzel \& Townes (1987) published their often referenced "enclosed mass" diagram which suggested the presence of a central dark mass of $2 \cdot 10^{6} M_{\odot}$ in the center of the Galaxy and since then it has always been discussed how significant this result is (e.g. Sellgren 1989, Saha et al. 1996). But now K-band spectroscopy of the $\mathrm{GC}$ region (Haller et al. 1996, Genzel et al. 1996) has confirm the presence of a dark mass of $M_{\bullet}=2.5 \cdot 10^{6} M_{\odot}$ within $0.1 \mathrm{pc}$ of $\mathrm{Sgr} \mathrm{A}^{*}$ with a $>6 \sigma$ significance. Even more fascinating is the detection of proper motion for some of the stars in the very vicinity of $\mathrm{Sgr}$ $A^{*}$ with Speckle imaging (Eckart \& Genzel 1996a\&b). This combined with the velocity dispersions determines the velocity field of the stars in three dimensions and one infers a mass density of $6.5 \cdot 10^{9} M_{\odot} \mathrm{pc}^{-3}$. The accuracy of those Speckle images was impressively confirmed at a recent conference by an independent $\mathrm{K}$-band speckle image with 50 mas resolution obtained with the Keck telescope (Klein et al. 1996). The race for the most accurate mass determination has just begun: with more 8 and 10 meter telescopes doing NIR Speckle imaging and HST dedicating a lot of its NICMOS time to the Galactic Center (Rieke 1996) we can expect that these results will improve even more dramatically. Very interesting in this respect is the announcement of a detection of a high proper motion star $(\sim 1500 \mathrm{~km} / \mathrm{sec})$ just a fraction of an arcsecond away from Sgr A* (Eckart \& Genzel 1996a\&b). For the cluster of faint NIR stars immediately surrounding $\operatorname{Sgr} \mathrm{A}^{*}$ the Keplerian motion around the black hole at a distance of 0.1 arcsecond is $1600 \mathrm{~km} / \mathrm{sec}$ and one would expect to see a proper motion of the order $40 \mathrm{mas} / \mathrm{yr}$ for the nearest stars. This should be well detectable within the very near future and in the extreme limit could push the central dark mass density to almost $10^{13} M_{\odot} / \mathrm{pc}^{3}$. The disovery of a cusp in the stellar distribution could also increase the lower limit on the mass of the radio source Sgr A* derived from the absence of its proper motion (Backer 1996) from several $100 M_{\odot}$ to something more like $1000 M_{\odot}$. Given that prospect and the already stunning success NIR Speckle imaging had in the Galactic Center, it is really surprising that so little time and attention is given to NIR Speckle imaging programs with Keck - if that would have different the matter could have been settled by now. 


\subsection{Spectrum}

While the mass determination for the Galactic Center is getting more and more exciting every year, the spectrum of $\mathrm{Sgr} \mathrm{A}^{*}$ is getting more and more disappointing. This source has a long history of detections at wavelengths other than the radio, none of which survived very long. The latest frustration stems from efforts to accurately align the radio and the optical reference frame using Masers associated with stars (Menten et al. 1996). This work seems to indicate that none of the sources detected in NIR Speckle images coincide with the radio source Sgr $A^{*}$. There is a claim now of a detection of a source at $8.7 \mu \mathrm{m}$ (Stolovy et al. 1996) within $0.3^{\prime \prime}$ of $\operatorname{Sgr~A}$ * which was not seen in earlier observations (Gezari 1996). This needs to be - and will be - checked by further observations with higher resolutions (e.g. with Keck, Morris et al., in prep.). Except for a possible weak ROSAT detection (Predehl \& Trümper 1994), there is currently no unambiguous evidence for a detection of $\mathrm{Sgr} \mathrm{A}^{*}$ at any higher frequency (Maeda et al. 1996).

Hence, the only informations we can use right now are radio data. There is some confusion in the current literature about the actual spectrum of Sgr A*. Duschl \& Lesch (1994) compiled an average spectrum from the literature and claimed a $\nu^{1 / 3}$ spectrum indicative of optically thin emission from monoenergetic electrons.

However, in simultaneous multi-frequency VLA observations (Wright \& Backer 1993) the actual spectrum was bumpy and the spectral index varied between $\alpha=0.19-0.34\left(S_{\nu} \propto \nu^{\alpha}\right)$. Morris \& Serabyn (1996) published a more recent spectrum that was a smooth powerlaw with $\alpha=0.25$ below $100 \mathrm{GHz}$. Moreover, in 24 simultaneous observations at 2.7 and $8 \mathrm{GHz}$ between 1976 and 1978 the spectral index varied between $\alpha_{2.7 / 8}=0.08$ and $\alpha_{2.7 / 8}=0.56$ with a mean value of $\alpha_{2.7 / 8}=0.28 \pm 0.01$ (Brown \& Lo 1982). This data is apparently affected (steepened) by the low-frequency turnover of the spectrum around $1 \mathrm{GHz}$ which seems to be variable in frequency with a timescale of several years: in 1976 the average 2.7 to $8 \mathrm{GHz}$ spectral index was $\alpha_{2.7 / 8}=0.34$ wile in the consecutive two years it was significantly lower at $\alpha_{2.7 / 8}=0.23$. More recent observations with the GBI showed a steady decline at low frequencies with a $2.7 \mathrm{GHz}$ flux now around $100 \mathrm{mJy}$ and a basically unchanged $8 \mathrm{GHz}$ flux (T. Minter, priv. comm.) yielding a spectral index around 1.5. At shorter wavelengths the spectral index is markedly flatter. The simultaneous VLA observations of Sgr A* in 1990/91 by Zhao et al. (1992) indicate an average spectral index of $\alpha_{8.4 / 15} \simeq 0.22$ between 8.4 and $15 \mathrm{GHz}$ and $\alpha_{15 / 22} \simeq 0.15$ between 15 and $22.5 \mathrm{GHz}$. The spectrum changes once more if one looks at higher frequencies: the average flux at 22.5 $\mathrm{GHz}$ is $1 \mathrm{Jy}$ (Zhao et al. 1992) while it was $2.9 \pm 0.3 \mathrm{Jy}$ at $230 \mathrm{GHz}$ in the years 1987-1994 (Zylka et al. 1995) yielding $\alpha_{22 / 230}=0.45 \pm 0.5$.

So why am I mentioning all these number? Mainly because we had heated debates in recent years about this spectrum. As Sgr A* may be an archetype for compact radio cores in other galaxies, extreme care has to go into the interpretation of its spectrum - most likely any other radio core will have much less information available, and any error we make here will propagate into endless space.

In conclusion, if we want to describe the radio spectrum of $\operatorname{Sgr} \mathrm{A}^{*}$ we have to deal with at least four regimes: 1) a slowly variable low frequency 
turnover somewhere below $5 \mathrm{GHz}, 2$ ) a fairly flat powerlaw in the $\mathrm{cm}$ regime with a spectral index around $0.2,3$ ) an excess towards the sub-mm with much larger spectral index, and 4) a cut-off towards the IR (see Morris \& Serabyn 1996). Therefore it is not possible to describe the spectrum from $\mathrm{cm}$ to submm wavelengths with a single powerlaw.

\subsection{Size}

Concerning the size of $\operatorname{Sgr} \mathrm{A}^{*}$, there have been no new developments in the last years. The smallest size found with VLBI so far is $<1$ AU at $3 \mathrm{~mm}$ (Rogers et al. 1994, Doeleman et al. 1995) consistent with scatter broadening even at these frequencies. So far the weak extended component found by Krichbaum et al. (1993) has not been seen again and recent measurements of the different groups at $3 \mathrm{~mm}$ are in reasonable agreement. This is not necessarily surprising as Sgr $A^{*}$ is strongly variable with frequent bursts on a timescale of months (Zhao et al. 1992) which in AGN are often associated with the expulsions of blobs. Continued VLBI monitoring at $7 \mathrm{~mm}$ and $3 \mathrm{~mm}$, possibly linked to flux monitoring, is therefore necessary and further 3 and even $1 \mathrm{~mm}$ VLBI experiments are already being evaluated (Krichbaum et al., in prep.).

\subsection{Imaging a black hole with submm VLBI}

As pointed out in Falcke (1996a,b), the existence of a submm excess in the $\mathrm{Sgr}$ A* spectrum (see above) indicates that synchrotron-self absorption is present between 100 and $300 \mathrm{GHz}$ and this requires a source size of $\sim 2$ Schwarzschildradii $\left(R_{\mathrm{s}}\right)$. For comparison, I note that a Schwarzschild black hole has an innermost, marginable stable orbit of $3 R_{\mathrm{s}}$ while an extreme Kerr black hole has $0.5 R_{\mathrm{s}}$. Hence, with the increasingly more accurate mass and spectrum determination of Sgr $\mathrm{A}^{*}$ we might eventually be able to give lower limits for the spin of the black hole. Even more exciting is the prospect that we might actually be able to image the horizon of the black hole and, in contrast to his pessimism, see some of the effects R. Wehrse discussed during this conference. A black hole embedded in front of a luminous object (or embedded in a transparent synchrotron-emitting plasma blob) would indeed appear as a black hole. The shape of this "hole" was calculated by Bardeen (1973, Fig. 6) which in the case of Sgr A* should have a diameter of 25 micro-arcsecond. Observations with such a beamsize are in principle possible with global mm- and submm-VLBI and hence $\mathrm{Sgr} \mathrm{A}^{*}$ should serve as a strong motivation for developing these techniques to their full potential.

\section{Sgr $A^{*}$ - Theory}

\subsection{Low- and high-frequency cut-off}

While describing the spectrum of $\mathrm{Sgr} \mathrm{A}^{*}$ is difficult, modeling it is even more so. The low-frequency turn-over can, however, be understood fairly well by freefree absorption by the H II region Sgr A West in which Sgr A* is presumably embedded (see e.g. Beckert et al. 1996). Of course an intrinsic cut-off, e.g. due to self-absorption, can not be excluded, but the effects of free-free absorption are readily seen already at larger scales (Pedlar et al. 1989). The variability time scale of the cut-off frequency of a few years, requires that a substantial 
amount of the absorption happens very close to the center at a distance of roughly $10^{16} \mathrm{~cm}$ (assuming Kepler velocities). This scale is approximately the scale of the closest NIR stars and also the scale derived for the location of the refractive interstellar scintillation (RISS) which Zhao et al. (1989) suggested is responsible for the variability of $\mathrm{Sgr} \mathrm{A}^{*}$ at lower frequencies. But it is unlikely that the same material also produces the scatter-broadening of $\operatorname{Sgr} \mathrm{A}^{*}$, as one sees similar amounts of broadening in $\mathrm{OH}$ Masers throughout the central $100 \mathrm{pc}$ (Frail et al. 1994).

The high-frequency cut-off of $\mathrm{Sgr} \mathrm{A}^{*}$ in the sub-mm is usually explained by an absence of high-energy electrons, either because of a thermal (Melia 1992, Narayan et al. 1995) or a quasi-monoenergetic electron distribution, the latter being due to either magnetic reconnection (Duschl \& Lesch 1994), or as a consequence of hadronic processes (Falcke 1996a).

\subsection{Sgr A*: Accretion models}

The first coherent attempt to understand Sgr A* was made by Rees (1982) where he suggested that $\mathrm{Sgr} \mathrm{A}^{*}$ is a black hole accreting directly from the ISM. He pointed out that, as the infall timescale is short compared to the cooling timescale, a hot ion supported torus should form around $\mathrm{Sgr} \mathrm{A}^{*}$ and that the synchrotron radiation would be produced by hot thermal electrons. The only crudely estimated spectral index for this model was between $\alpha=0.4$ and $\alpha=1.3$ and hence far too high.

In recent years quite a few modern variations of the Rees model have appeared in the literature. Melia (1992, 1994, \& 1996) and Ozernoy (1992) explored the possibility that $\mathrm{Sgr} \mathrm{A}^{*}$ accretes from nearby stellar sources rather than from the ISM. However, as indicated in Falcke (1996a) to properly determine the wind accretion rate one will have to take the proper position (see Melia 1996) and angular velocity of the stars into account, this is especially important, as Eckart \& Genzel (1996) and Genzel et al. (1996) report that the He I stars seem to form a counterrotating entity.

The Melia model and standard accretion models, like the "starving disk" (Falcke et al. 1993a, Falcke \& Heinrich 1994), which have also been proposed now face some serious problems with the NIR fluxes: the observational limits are getting too low. A remedy would be an obscuring (micro-)torus around Sgr $\mathrm{A}^{*}$, and there are some indications in the putative ROSAT counterpart to Sgr $\mathrm{A}^{*}$ that there indeed is intrinsic absorption (Predehl \& Trümper 1994, Predehl \& Zinnecker 1996) in Sgr A*. Despite this weak sign of hope, Melia and I have joined forces to save our respective models from the advent of even more sensitive observations and have suggested that a fossil accretion disk in Sgr A* could capture a large fraction of the incoming wind already at larger radii. This could reduce the amount of optical and NIR emission substantially (Falcke \& Melia 1996).

As an alternative model Narayan et al. (1995) suggested an advection dominated disk model for the Galactic Center which has a concept very similar to the Rees model with wind accretion. The main plus of this model is that, because $>99.9 \%$ of the energy is swallowed by the black hole, it predicts very low NIR and UV luminosities (but with further decreasing limits on $\mathrm{Sgr} \mathrm{A}^{*}$ even these could become too high). On the other hand, the model only fits the submm 
bump but not the cm-to-mm radio spectrum and the assumed black hole mass of $M_{\bullet}=5 \cdot 10^{5} M_{\odot}$ is a factor 5 too small. The mass is a critical parameter for the submm spectrum and it is quite likely, that for a higher mass the peak in the spectrum would shift to lower frequencies, making the fit even worse.

\subsection{Jet-models for Sgr $A^{*}$}

Early on in the discussion, Reynolds \& McKee (1980) argued that Sgr A* must be an outflow because its energy density $u$ is so large that it cannot be constrained by gravity. For gravitational confinement of a plasma bubble with radius $R$, we would require

$$
G M_{\bullet} \rho / R>u \text {. }
$$

Synchrotron theory and equipartition arguments give us a lower limit for the energy density in the plasma for a fluxdensity $S_{\nu}$ at a frequency $\nu$. Using the above equation this translates into an upper limit for the size of Sgr A*

$$
R<3.5 \cdot 10^{13} \mathrm{~cm}\left(S_{\nu} / \mathrm{Jy}\right)^{1 / 10}(\nu / 5 \mathrm{GHz})^{-3 / 10}\left(M_{\bullet} / 2.5 \cdot 10^{6} M_{\odot}\right)^{7 / 10}
$$

and a lower limit for the brightness temperature

$$
T_{\mathrm{b}}>2.4 \cdot 10^{12} \mathrm{~K}\left(S_{\nu} / \mathrm{Jy}\right)^{4 / 5}(\nu / 5 \mathrm{GHz})^{-7 / 5}\left(M_{\bullet} / 2.5 \cdot 10^{6} M_{\odot}\right)^{-7 / 5}
$$

Such a high $T_{\mathrm{b}}$ would violate the Compton limit and therefore $\operatorname{Sgr} \mathrm{A}^{*}$ can not be gravitationally bound. Some recent models violate the condition in Eq. 1 by an order of magnitude and thus would require well ordered magnetic fields to confine the plasma, but that would also imply large polarization which is not seen.

Therefore, we have developed the jet/disk symbiosis model for the Galactic Center and other compact radio cores (Falcke et al. 1993b, Falcke \& Biermann 1995 , Falcke 1996c). The basic idea is that a jet flow is most likely coupled to an accretion disk which determines the jet power. For black holes the escape speed is relativistic and it is likely that the sound speed as well as the bulk velocity of the flow is also relativistic. Given a certain fraction of the accretion power which is channeled into the jet (here $50 \%$ of the dissipated energy) and assuming an equipartition situation, one can calculate the synchrotron radiation produced by the jet, yielding a flat to inverted spectrum. For many sources, e.g. like radioloud quasars (Falcke et al. 1995) and $\mathrm{Sgr} \mathrm{A}^{*}$, all equipartition factors have to be close to unity to get the very efficiently produced radio emission observed. Geometry, velocity field, and energy densities are self-consistently determined by assuming a free, supersonic flow which is slowly accelerated by its own pressure gradient.

For the Galactic Center it turned out, that a very small accretion rate would be enough to produce the $1 \mathrm{Jy}$ source $\mathrm{Sgr} \mathrm{A}^{*}$, with a size small enough not to be resolved with the current VLBI experiments. Alas, as pointed out above, $\mathrm{Sgr} \mathrm{A*}$ is not an ideal source anymore to test the validity of this model - too many parameters are still unconstrained. Therefore, we have applied this model to other galactic nuclei, like M81, where it perfectly fits the observed data. In the following, I want to discuss several other Galaxies with compact radio cores, that might be directly relevant to the discussion in the Galactic Center. 


\section{Jets and compact radio cores in nearby Galaxies}

\subsection{NGC 4258}

One of the most famous galaxies in recent years was NGC 4258 . VLBI observations of $\mathrm{H}_{2} \mathrm{O}$ masers in this LINER galaxy have revealed a thin Keplerian molecular disk around the nucleus with a central dark mass of $M_{\bullet}=3.6 \cdot 10^{7} M_{\odot}$ (Miyoshi et al. 1995). Earlier VLA observations (Turner \& Ho 1994) showed a three $\mathrm{mJy}$, compact radio core with inverted spectrum. At this conference, Herrnstein et al. (1996) presented VLBA observations of NGC 4258 at $1.3 \mathrm{~cm}$ where they found a variable continuum source with a flux of 1-6 mJy which was 0.4-0.8 mas offset from the dynamical center of the masing disk in the direction of the rotation axis. Once more this galaxy proves to be an important laboratory because it is the only case where we are able to determine the dynamical center of a disk with respect to the radio core. NGC 4258 seems to confirm the jet model in which, because of its conical geometry, the innermost part of the jet close to the center becomes synchrotron self-absorbed. If the radio core were for example an accretion disk atmosphere like in some $\mathrm{Sgr} \mathrm{A}^{*}$ models, one would expect to see radio emission centered on the nucleus.

We can now apply the jet-disk symbiosis model as used for Sgr A* and M81* (Falcke 1996c) without any modifications to NGC 4258. As the inclination angle of the disk is already fixed at $i \sim 83^{\circ}$ by the VLBA maser observations, the model has only two adjustable parameters, the accretion disk luminosity and the typical Lorentz factor $\gamma_{\mathrm{e}}$ of the radiating electrons, for two observed data points (offset and flux). It predicts a flux of $3 \mathrm{mJy}$ and an offset of 0.4 mas if the accretion disk luminosity of NGC 4258 is $L_{\text {disk }} \sim 4 \cdot 10^{41} \mathrm{erg} / \mathrm{sec}$ and $\gamma_{\mathrm{e}} \sim 700$. The latter value is relatively large (e.g. three times larger than estimated for $\mathrm{M} 81^{*}$ ), but would become lower if the observed offset is overestimated, for example because NGC 4258 expels radio blobs more frequently than it was observed. On the other hand, the accretion disk luminosity - here solely derived from the radio properties alone - is well within current estimates: Stüwe et al. (1992), trying to fit observed line ratios, postulate a hidden UV source of $L \sim 3 \cdot 10^{41} \mathrm{erg} / \mathrm{sec}$, and Wilkes et al. (1995) estimate $L \sim 10^{42} \mathrm{erg} / \mathrm{sec}$ from light scattered off dust. This luminosity is also consistent with the accretion rated estimated by Neufeld \& Maloney (1995) for the molecular disk. Hence, we can in principle construct a self-consistent, quantitative model, where the accretion rate measured in the masing disk, feeds a black hole and an inner disk that produces x-rays, UV emission, and a radio jet plus counterjet, which in turn illuminate the disk and provide seed photons for the masers. Consequently, there is currently no need for an advection dominated disk in NGC 4258 as proposed by Lasota et al. (1996). The jet/disk model can also explain the large scale emission-line jet in NGC 4258 , since its kinetic power is of the order $10^{42} \mathrm{erg} / \mathrm{sec}-$ as derived from the mass $\left(2 \cdot 10^{6} M_{\odot}\right)$ and velocity $(\sim 2000 \mathrm{~km} / \mathrm{sec})$ of the emission-line gas (Cecil et al. 1995). One of the conclusions of the jet/disk symbiosis model always was that jet and disk have comparable powers and NGC 4258 seems to confirm this.

\subsection{NGC 3079}

We can further check the same model with yet another Megamaser, namely the LINER NGC 3079. This highly inclined galaxy $\left(i=83^{\circ}, D=18 \mathrm{Mpc}\right)$ has huge 
radio and optical lobes (superbubbles) perpendicular to the plane of the galaxy with a power of the order $\dot{E}_{\text {kin }} \sim 6 \cdot 10^{42} \mathrm{erg} / \mathrm{sec}$ (Veilleux et al. 1994), however, at present it is not clear whether the lobes are due to starbursts or a central engine. The nuclear IR luminosity of NGC 3079 is $\sim 3 \cdot 10^{43} \mathrm{erg} / \mathrm{sec}$ and we assume that the luminosity of the AGN is of similar order. In the very nucleus we find a compact, milliarcsecond radio core with a flux of $\sim 65 \mathrm{mJy}$. And indeed for an $L_{\text {disk }} \sim 3 \cdot 10^{43} \mathrm{erg} / \mathrm{sec}$ the jet/disk symbiosis model predicts the right size and flux of the compact radio core, if the electron Lorentz factor is $\gamma_{e}=275$ and the inclination $\sim 75^{\circ}$. The high inclination agrees well with the high inclination of the galaxy (which, however, need not always be related) and the fact that we see maser emission. Most importantly, this means that the relatively high luminosity of the radio core requires a powerful central engine and a powerful radio jet which in fact would be sufficient to drive the lobes without the help of a starburst-driven superwind. In summary, this galaxy could in many details be very similar to NGC 4258 with one important difference: if we compare the ratio $R_{1 / c}$ between the lobe and the core radio flux in both galaxies, we find that NGC 4258 has $R_{1 / \mathrm{c}} \sim 200$, while NGC 3079 has only $R_{1 / \mathrm{c}} \sim 5$. The best explanation for this discrepancy is the different orientation of the lobes: in NGC 4258 the jet has to go through the disk of the galaxy and the ISM, which may increase the radiative efficiency, while in NGC 3079 the lobes go out of the plane of the galaxy with presumably much less interaction.

\subsection{NGC 6500}

One important assumption in the modeling so far was that all these radio jets start out with relativistic speeds and bulk Lorentz factors of $\gamma_{j} \sim 2-3$ because mildly relativistic speeds are required if the jets want to escape from the vicinity of the black hole. A necessary consequence would then be relativistic boosting in systems seen face on. One such case could indeed be NGC $6500\left(i=34^{\circ}\right.$, $D=63 \mathrm{Mpc}$ ), which has a $200 \mathrm{mJy}$ radio core with a size of $\sim 3$ mas (Jones et al. 1981) and large-scale lobes as in NGC 3079 (Unger et al. 1989). The nuclear luminosity of this galaxy cannot be much larger than in NGC 3079, since its IR luminosity is comparable to NGC 4258 , yet its radio core is much brighter. Nor surprisingly, the jet/disk model predicts that the inclination angle for the radio jet has to be around $25-30^{\circ}$ in order to explain the bright radio core. This would mean, that we are looking right into the boosting cone of the jet and one could consider this galaxy a low-luminosity equivalent to a Blazar.

\section{Seyferts and radio-weak quasars}

So far we have discussed only LINER galaxies with compact radio cores. But what is the situation in Seyfert galaxies? Surprisingly, only $10 \%$ of Seyfert galaxies show flat spectrum cores (deBruyn \& Wilson 1978), and Sadler et al. (1995) found that the pc scale cores seen in many Seyfert galaxies probably have a steep spectrum. Yet, many Seyfert galaxies apparently have large-scale jets (Ulvestad \& Wilson 1989, and refs. therein), and one of the important results of HST for Seyfert galaxies is that the emission-line region (NLR) of Seyferts can be substantially modified by the impact of the radio jet. The best examples are perhaps MK 573 (e.g. Capetti et al. 1996) and ESO 428-G14 (Falcke et 
al. 1996b). The former has a NLR with bow-shock like features around radio hot-spots, while the NLR of the latter consists of thin narrow strands of emission line gas, which apparently wrap around a radio jet and form a figure eight on one side, suggesting helical motion. In those two cases it is obvious that the jet dominates and shapes the emission-line gas of these Seyfert galaxies.

Conventional wisdom suggests that Quasars are the more luminous counterparts to Seyfert galaxies and we can ask how important are jets therein? The answer is simple for radio-loud quasars which harbor luminous compact radio cores and powerful relativistic jets. However, only $10 \%$ of all quasars are radioloud, while the rest is radio-quiet - not completely quiet though, because many of them have compact, or diffuse extended radio emission at the milli-Jansky level and below. In a few cases Kellerman et al. (1994) found bipolar structures which they interpreted as radio jets. Moreover, we have recently investigated the properties of a mysterious class of radio-intermediate quasars (RIQ, Falcke et al. 1996a). These are flat-spectrum core-dominated quasars, with radio luminosities (normalized to the optical luminosity) below those of typical, lobedominated steep spectrum quasars, and they do not have any extended emission. Hence, these sources can not be relativistically boosted radio-loud quasars (as usually assumed for core-dominated quasars), instead, many arguments suggest that these quasars are preferentially oriented, relativistically boosted, radio-quiet quasars. The relatively large fraction of RIQs then also implies that basically all radio-weak quasars have such relativistic jets in their nuclei (but are pointing away from us).

\subsection{Are LINERs radio-loud?}

In summary, there is a lot of evidence now, that jets are integral and dynamically important parts of Seyfert galaxies and radio-quiet quasars as well. For some reasons, however, their radiative efficiency is much lower than in radio-loud quasars. In this respect it is interesting to note that many of the galaxies with prominent, compact radio cores are LINERs, like all the galaxies discussed in this paper, and the model used here to explain their radio-cores is exactly the same as for radio-loud quasars. Unfortunately, there is currently no good statistical data base to back this claim, but it might be an interesting question to ask whether the central engine in some LINERs are the counterparts to radio-loud quasars, while the AGN in Seyfert galaxies are the counterparts to radio-weak quasars at lower accretion rates.

\section{Conclusions}

The evidence that the Galactic Center harbors a supermassive black hole of mass $\sim 2.5 \cdot 10^{6}$ is now close to overwhelming. The compact radio core Sgr $\mathrm{A}^{*}$ which is probably associated with this supermassive black hole, does not appear to be a very unusual object compared to the radio cores in the nuclei of other galaxies, unusual are only its optical/NIR properties (or better the lack thereof). Advection dominated disk models may explain the low radiative efficiency, but fail to properly explain the radio spectrum, the jet/disk model on the other hand can explain the radio spectrum fairly well, yet predicts too much optical/NIR flux. These problems do not necessarily exclude any of these 
models yet, but could mean that we are still missing a critical (observational) piece in the Galactic Center puzzle.

Personally, I believe that the jet model still has the most merits, since it works well for compact radio cores in all other galaxies. The properties of all the galaxies discussed here (Milky Way, M81, NGC 4258, NGC 3079, \& NGC 6500 ), i.e. the fluxes and sizes of their radio cores, can be explained by one model with basically three input parameters: the accretion rate, the inclination to the line of sight, and (as the only fudge-factor), the typical Lorentz factor of the relativistic electrons. In some of these galaxies, e.g. like NGC 4258, and of course all radio-loud quasars and radio galaxies, the association between jet and compact radio core is almost indisputable. Therefore, until demonstrated otherwise, there is no reason to believe that in sources like $\mathrm{Sgr} \mathrm{A}^{*}$ where no large scale jet is yet detected, the radio cores are anything but the bases of radio jets powered by accretion onto a massive, compact object.

As Livio (1996) pointed out there is hardly any class of sources left where accretion is not accompanied by a bipolar outflow. And as discussed in this paper we find compact radio-cores or powerful large-scale jets in many extragalactic sources, like LINERs, Seyferts, and even radio-quiet quasars. This makes the jet phenomenon a truly universal phenomenon.

Acknowledgments. I acknowledge support from NASA under grants NAGW3268 and NAG8-1027.

\section{References}

Backer D.C. 1996, in "Unsolved Problems of the Milky Way", IAU Symp. 169, L. Blitz \& Teuben P.J. (eds.), Kluwer, Dordrecht, p. 193

Bardeen, J.M. 1973, in: "Black Holes", DeWitt, C. \& DeWitt, B.S. (eds.), Gordon and Breach, Paris, p. 215

Beckert T., Duschl W.J., Mezger P.G., \& Zylka R. 1996, A\&A 307, 450

Brown R.L., \& Lo K.Y. 1982, ApJ 253, 108

Capetti, A., Axon, D.J., Macchetto, F., Sparks, W.B., \& Boksenberg, A. 1996, ApJ, in press

Cecil G., Wilson A.S., \& De Pree C. 1995, ApJ 440, 181

deBruyn A.G., \& Wilson A.S. 1978, A\&A 64, 433

Doeleman S., Rogers A.E.E., Bower G.C., Wright M.C.H.,\& Backer D.C. 1995, BAAS $187, \# 12.14$

Duschl W.J., \& Lesch H. 1994, A\&A 286, 431

Eckart, A., \& Genzel, R. 1996a, in: "The Galactic Center", ASP Conf. Series Vol. 102, R. Gredel (ed.), p. 196

Eckart, A., \& Genzel, R. 1996b, MNRAS, in press

Eckart, A., Genzel, R., Krabbe, A., Hofmann, R., van der Werf, P. P., \& Drapatz, S. 1992 , Nat 355,526

Falcke H., 1996a, in "Unsolved Problems of the Milky Way", IAU Symp. 169, L. Blitz \& Teuben P.J. (eds.), Kluwer, Dordrecht, p. 163 
Falcke H. 1996b, in: "The Galactic Center", ASP Conf. Ser. 102, R. Gredel (ed.), p. 453

Falcke H. 1996c, ApJ 464, L67

Falcke H., \& Biermann P.L. 1995, A\&A 293, 665

Falcke H., \& Heinrich O. 1994, A\&A 292, 430

Falcke H., \& Melia F. 1996, to appear in ApJ

Falcke H., Biermann P. L., Duschl W. J., \& Mezger P. G. 1993a, A\&A 270, 102

Falcke H., Mannheim K., \& Biermann P. L. 1993b, A\&A 278, L1

Falcke H., Malkan M., \& Biermann P.L. 1995, A\&A 298, 375

Falcke H., Patnaik A., \& Sherwood W. 1996, ApJ Letters, in press

Falcke H., Wilson A.S., Simpson C., \& Bower G.A. 1996, ApJ 470, L31

Frail, D.A., Diamond, P.J., Cordes, J.M., \& van Langevelde, H.J. 1994, ApJ, L43

Genzel R., Thatte N., Krabbe A., Kroker H., \& Tacconi-Garman L.E. 1996, ApJ, in press

Gezari, D. 1996, in "The Galactic Center", ASP Conf. Ser. 102, R. Gredel (ed.), 491

Haller J., Rieke, M., Rieke, G., Tamblyn, P. Close, L. \& Melia, F. 1995, ApJ 456, 194

Herrnstein J., Moran J.M., Greenhill L.J. et al. 1996, — this volume

Ho L.C., Filippenko A.V., \& Sargent W.L.W. 1995, ApJS 98, 477

Jones D.L., Sramek R.A., \& Terzian Y. 1981, ApJ 246, 28

Kellermann, K.I., Sramek, R., Schmidt, M., Green, R., \& Shaffer, D.B. 1994, AJ 108, 1163

Klein B.L., Ghez A.M., Morris M., \& Becklin E.E. 1996, in "The Galactic Center", ASP Conf. Ser. 102, R. Gredel (ed.), p. 228

Krichbaum T.P., Zensus J.A., Witzel A., et al. 1993, A\&A 274, L37

Lasota J.-P., Abramowicz M.A., Chen X., Krolik R., Narayan R., \& Yi I. 1996, ApJ 462, L141

Latvakoski H., Stacey G., Hayward T., \& Gull G. 1996, this volume

Livio M. 1996, - this volume

Maeda Y., Koyama K., Sakano M., Takeshima T., \& Yamauchi S. 1996, PASJ 48,417

Melia F. 1992, ApJ 387, L25

Melia F. 1994, ApJ 426, 677

Melia F., 1996, - this volume

Menten K.M., Reid M.J., Eckart A., \& Genzel R. 1996, ApJ Letters, in press

Morris M., \& Serabyn G. 1996, ARA\&A 34, in press

Miyoshi M., Moran J., Herrnstein J. et al. 1995, Nat 373, 127

Narayan R., Yi I., \& Mahadevan R. 1995, Nat 374, 623

O'Connel R.W., \& Dressel L.L. 1996, Nat 276, 374 
Ozernoy L. 1992, "Testing the AGN Paradigm", AIP Conf. 254, Holt, S.S. et al. (eds.), New York, p. 40,44

Predehl P., and Trümper J. 1994, A\&A 290, L29

Predehl P., \& Zinnecker H. 1996, in: "The Galactic Center", ASP Conf. Ser. 102, R. Gredel (ed.), p. 415

Rees M. 1982, in: "The Galactic Center", AIP Conf. 83, eds. Riegler \& Blandford, p. 166

Reynolds S.P., \& McKee C.F. 1980, ApJ 239, 893

Rieke M. 1996, GCNEWS 1, 3 (see http://www.astro.umd.edu/ gcnews)

Rogers A.E.E., Doeleman S., Wright M.C.H. et al. 1994, ApJ 434, L59

Sadler E.M., Slee O.B., Reynolds J.E., \& Roy A.L. 1995, MNRAS 276, 1373

Saha P., Bicknell G.V., \& McGregor P. 1996, ApJ 467, 636

Sellgren K. 1989, in: "The Center of the Galaxy", IAU Symp. 135, Morris M. (ed.), Kluwer, p. 477

Pedlar A., Anantharamaiah K.R., Ekers R.D. et al. 1989, ApJ 342, 769

Stolovy S.R., Hawyard T., \& Herter T. 1996, ApJ Letters, in press

Stüwe J.A., Schulz H., \& Hühnermann H. 1992, A\&A 261, 382

Turner J.L., \& Ho P.T.P. 1994, ApJ 421, 122

Ulvestad, J.S. \& Wilson, A.S. 1989, ApJ 343, 659

Unger S.W., Pedlar A., \& Hummel E. 1989, A\&A 208, 14

Veilleux S., Cecil G., Bland-Hawthorn J., Tully R.B., Filippenko A.V., \& Sargent W.L.W. 1994, ApJ 433, 48

Wehrse R. 1996, - this volume

Wright M.C.H., \& Backer D.C. 1993, ApJ 417, 560

Wrobel J.M., \& Heeschen D.S. 1984, ApJ 287, 41

Zylka R., Mezger P.G., Ward-Thomson D., Duschl W., \& Lesch H. 1995, A\&A 297, 83

Zhao J.-H., Ekers R.D., Goss W.M., Lo K.Y., \& Narayan R. 1989, in: "The Center of the Galaxy", IAU Symp. 135, Morris M. (ed.), Kluwer, p. 535

Zhao J.-H., Goss W.M., Lo K.Y., \& Ekers R.D. 1992, in: "Relationships Between Active Galactic Nuclei and Starburst Galaxies", ASP Conf. Ser. 31, A. Filippenko (ed.), p. 295 